\title{
Diffusion with Stochastic Resetting
}

\author{
Martin R. Evans ${ }^{(1,2)}$ and Satya N. Majumdar ${ }^{(2)}$ \\ (1) SUPA, School of Physics and Astronomy, University of Edinburgh, \\ Mayfield Road, Edinburgh EH9 3JZ, United Kingdom \\ (2) Univ. Paris-Sud, CNRS, LPTMS, UMR 8626, Orsay F-01405, France
}

\begin{abstract}
We study simple diffusion where a particle stochastically resets to its initial position at a constant rate $r$. A finite resetting rate leads to a nonequilibrium stationary state with non-Gaussian fluctuations for the particle position. We also show that the mean time to find a stationary target by a diffusive searcher is finite and has a minimum value at an optimal resetting rate $r^{*}$. Resetting also alters fundamentally the late time decay of the survival probability of a stationary target when there are multiple searchers: while the typical survival probability decays exponentially with time, the average decays as a power law with an exponent depending continuously on the density of searchers.
\end{abstract}

PACS numbers: 05.40.-a, 02.50.-r, 87.23.Ge

'Stochastic resetting' is a rather common process in everyday life. Consider searching for some target such as, for example, a face in a crowd or one's misplaced keys at home. A natural tendency is, on having searched unsuccessfully for a while, to return to the starting point and recommence the search. In this Letter we explore the consequences of such resetting on perhaps the most simple and common process in nature, namely, the diffusion of a single or a multiparticle system. We show that a nonzero rate of resetting has a rather rich and dramatic effect on the diffusion process.

The first major effect of resetting shows up in the position distribution of the diffusing particle. In the absence of resetting, it has the usual Gaussian distribution whose width grows diffusively $\sim \sqrt{t}$ with time. Upon switching on a nonzero resetting rate $r$ to its initial position, this time-dependent Gaussian distribution gives way to a globally current-carrying nonequilibrium stationary state (NESS) with non-Gaussian fluctuations, given in Eq. (2). The process of resetting manifestly violates detailed balance and thus provides an appealingly simple example of a NESS.

Resetting also has a profound consequence on the firstpassage properties of a diffusing particle. The study of first-passage problems and survival probabilities of diffusing particles arises in diverse subjects such as in reactiondiffusion kinetics, predator-prey dynamics 1], as well as in persistence in nonequilibrium systems [2]. Such problems are fundamental to nonequilibrium statistical mechanics as they involve irreversible processes not obeying detailed balance. Related models are also relevant to the study of search strategies in ecology or sampling techniques for the characterisation of complex networks. For example, intermittent searches involve diffusive motion combined with long range movements of the searcher and mimic the scan and relocation phases of foraging animals [3] 8].

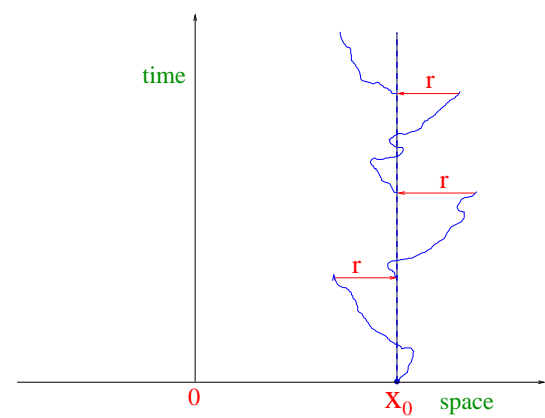

Figure 1. Schematic space-time trajectory of a one dimensional Brownian motion that starts at $x_{0}$ and resets stochastically to its initial position $x_{0}$ at rate $r$.

A well-studied problem is the mean time for a stationary target at the origin to be absorbed by a single diffusing particle (trap) or a team of diffusing traps distributed with uniform density. Many significant results, such as the fact that the mean time to find the target by a single diffusing particle diverges and that the survival probabilty of the target decays with time as a stretched exponential in the presence of a finite density of diffusing particles, are well-established when there is no resetting [1, 9, 10].

Our results, summarised in this paragraph, show that these statistical properties of the search for a stationary target by diffusing traps (searchers) are fundamentally altered when the searchers reset stochastically to their initial positions. In the case of a single diffusing trap, the mean time to find the target at the origin, given by Eq. (7), becomes finite in the presence of a non-zero resetting rate $r$ and as a function of $r$, has a minimum at a nontrivial value $r^{*}$ given by Eq. (8). Thus there is an optimal resetting rate that makes the target search most 
efficient. The survival probability of the target decays exponentially with nontrivial decay rate summarized in Eqs. (1213). For the case of mutiple traps distributed with uniform density $\rho$ and each diffusing independently with a diffusion constant $D$, the effect of resetting is even more dramatic. For example, in one dimension and in the absence of resetting, both the typical and the average survival probability of the target decay with time $t$ as a stretched exponential, $\sim \exp (-\lambda \rho \sqrt{D t})$, albeit with two different values of the decay constant $\lambda[9,10]$. In contrast, we find that when one switches on the resetting the typical survival probability of the target asymptoticaly decays faster with time, as an exponential, Eq. (26). On the other hand, the average survival probability decays more slowly asymptotically, as a power law with a nonuniversal exponent that varies continuously with density $\rho$, Eq. (23). This slower algebraic decay can be attributed to contributions arising from rare, extreme events.

We now present a derivation of our results. For simplicity, we focus below on the one-dimensional case, though generalization to higher dimensions is straightforward and will be mentioned at the end. To begin with we consider the simple case of a single particle diffusing in one dimension with diffusion constant $D$ and stochastically resetting to its initial position at a constant rate $r$ (see Fig. 1). The Master equation for $p\left(x, t \mid x_{0}\right)$, the probability that the particle is at $x$ at time $t$, having begun from $x_{0}$ at time 0 , simply reads

$$
\frac{\partial p\left(x, t \mid x_{0}\right)}{\partial t}=D \frac{\partial^{2} p\left(x, t \mid x_{0}\right)}{\partial x^{2}}-r p\left(x, t \mid x_{0}\right)+r \delta\left(x-x_{0}\right)
$$

with initial condition $p(x, 0)=\delta\left(x-x_{0}\right)$. The second and third terms on the rhs and represent a negative probability flux $r p\left(x, t \mid x_{0}\right)$ out of each point $x$ and a corresponding positive probability flux into $x_{0}$ which sums to $r$. It is easy to show that in the long-time limit the particle attains a stationary distribution

$$
p_{\text {st }}\left(x \mid x_{0}\right)=\frac{\alpha_{0}}{2} \exp \left(-\alpha_{0}\left|x-x_{0}\right|\right)
$$

where $\alpha_{0}=\sqrt{r / D}$ is an inverse length scale corresponding to the typical distance diffused by the particle between resets. The distribution (2) is evidently nonGaussian, with a cusp at $x=x_{0}$. Since resetting creates a source of probabality at $x_{0}$ while probability is lost from all $x \neq x_{0}$, there is circulation of probability even at long times, making (2) a non-equilibrium stationary state.

The result (2) has a simple and appealing interpretation in terms of a renewal process. The time evolution of the diffusive particle (see Fig. (11) is indeed a renewal process, where each resetting event to $x_{0}$ renews the process at a Poisson rate $r$ and between two consecutive renewal events, the particle undergoes free diffusion. Consider the particle at some fixed time $t$. If $\tau$ denotes the time elapsed since the last renewal, the position distribution is simply Gaussian, $e^{-\left(x-x_{0}\right)^{2} / 4 D \tau} / \sqrt{4 \pi D \tau}$. Clearly $0 \leq \tau \leq t$ is a random variable and for large $t$, $\tau$ is simply exponentially distributed, $\operatorname{Prob}(\tau)=r e^{-r \tau}$. Thus, for large $t$, the stationary position distribution of the particle is obtained by averaging over $\tau$

$$
p_{\mathrm{st}}\left(x \mid x_{0}\right)=\int_{0}^{\infty} d \tau r e^{-r \tau} \frac{e^{-\left(x-x_{0}\right)^{2} / 4 D \tau}}{\sqrt{4 \pi D \tau}}
$$

which precisely yields the result in (2).

We now turn to the first-passage properties through the origin of the diffusing particle in the presence of resetting. The origin can be thought of as a stationary target which is absorbed when the diffusing particle, starting initially at $x_{0}>0$ at $t=0$, hits the origin for the first time. The diffusing particle also resets to its initial position $x_{0}$ at a constant rate $r$. To solve the first-passage problem, it is advantageous to use the backward Master equation approach where one treats the initial position as a variable. Hence, we first set the initial position to be at $x>0$, different from the resetting position $x_{0}$, solve the problem with arbitrary $x$ and $x_{0}$ and eventually set $x=x_{0}$. Let $Q(x, t)$ denote the survival probability or persistence of the target up to time $t$ (it also depends implicitly on the resetting position $x_{0}$ ). This is then also the probability that the diffusing particle, starting at $x$, has not hit the origin up to time $t$. The backward Master equation (where the initial position $x$ is the variable) then reads

$$
\frac{\partial Q(x, t)}{\partial t}=D \frac{\partial^{2} Q(x, t)}{\partial x^{2}}-r Q(x, t)+r Q\left(x_{0}, t\right)
$$

with boundary and initial conditions $Q(0, t)=0$, $Q(x, 0)=1$. The second and third terms on the rhs correspond to the resetting of the initial position from $x$ to $x_{0}$, which implies a loss of probability from $Q(x, t)$ and a gain of probability to $Q\left(x_{0}, t\right)$. The Laplace transform $q(x, s)=\int_{0}^{\infty} \mathrm{d} t \mathrm{e}^{-s t} Q(x, t)$ satisfies

$$
D \frac{\partial^{2} q(x, s)}{\partial x^{2}}-(r+s) q(x, s)=-1-r q\left(x_{0}, s\right)
$$

which can be exactly solved by noting that the general solution is $q(x, s)=A \mathrm{e}^{\alpha x}+B \mathrm{e}^{-\alpha x}+\left(1+r q\left(x_{0}, s\right)\right) /(r+s)$ with $\alpha=\sqrt{(r+s) / D}$. Now $q(x, s)$ remains finite as $x \rightarrow \infty$ therefore $A=0$. The condition $q(0, s)=0$ fixes the constant $B=-\left(1+r q\left(x_{0}, s\right)\right) /(r+s)$ and $q\left(x_{0}, s\right)$ is determined self-consistently as

$$
q\left(x_{0}, s\right)=\frac{1-\exp \left(-\alpha x_{0}\right)}{s+r \exp \left(-\alpha x_{0}\right)} .
$$

The mean first-passage time, $T\left(x_{0}\right)$, may be obtained from $T\left(x_{0}\right)=-\int_{0}^{\infty} \mathrm{d} t t \frac{\partial Q\left(x_{0}, t\right)}{\partial t}=q\left(x_{0}, s=0\right)$ which yields

$$
T\left(x_{0}\right)=\frac{1}{r}\left(\exp \left(\alpha_{0} x_{0}\right)-1\right)
$$


where we recall $\alpha_{0}=\sqrt{r / D}$. The first point to note is that $T$ is finite for $r>0$ and diverges as $r \rightarrow 0$ as $T \sim r^{-1 / 2}$, which recovers the well-known result that the mean time for a diffusive particle to reach the origin (in the absence of resetting) is infinite. Also $T$ diverges as $r \rightarrow \infty$, the explanation being that as the reset rate increases the diffusing particle has less time between resets to reach the origin.

Now consider $T$ as a function of $r$ for a fixed $x_{0}$. Since $T$ diverges as $r \rightarrow 0$ and $r \rightarrow \infty$ it is clear that there must be a minimum of $T$ with respect to $r$ at an optimal value $r^{*}$. We consider the dimensionless variable $z=$ $\alpha_{0} x_{0}$, the ratio of the distance of the intial site from the target to the length diffused between resets, with which our results may be simply expressed. Then the condition for the minimum, $\mathrm{d} T / \mathrm{d} r=0$, reduces to the transcendental equation

$$
\frac{z^{*}}{2}=1-\mathrm{e}^{-z^{*}}
$$

which has a unique non-zero solution $z^{*}=1.59362 \ldots$. Thus, there is an optimal resetting rate $r^{*}=\left(z^{*}\right)^{2} D / x_{0}^{2}$ in one dimension that minimizes the search time to find the target at the origin.

It is difficult to invert the Laplace transform (6) explicitly for all parameters. However, one can deduce the large $t$ asymptotic behaviour for fixed parameters $r, x_{0}$. Generally this is determined by the singularity, in the complex $s$ plane, of $q\left(x_{0}, s\right)$ with largest real part. From (66) there will be a pole in $q\left(x_{0}, s\right)$ when

$$
s+r \mathrm{e}^{-\alpha x_{0}}=0
$$

which may be rewritten as $s=r(u-1)$ where $u$ satisfies

$$
u=1-\exp \left(-u^{1 / 2} z\right) .
$$

An addition to the trivial solution $u=0$ (which corresponds to the branch point at $s=-r$ ) there is exactly one non-trivial solution $u_{0}$ of (10) where $0<u_{0}<1$. Thus $q\left(x_{0}, s\right)$ has a simple pole at $s_{0}=-r\left(1-u_{0}\right)$ the residue of which determines the asymptotic behaviour as

$$
Q\left(x_{0}, t\right) \simeq \mathrm{e}^{s_{0} t} \frac{2 u_{0}^{3 / 2}}{z u_{0}+2 u_{0}^{1 / 2}-z} .
$$

Different limiting values of $u_{0}$ and hence $s_{0}$ can be deduced. For $z \ll 1, u_{0} \simeq z^{2}$ and $s_{0} \simeq-r+r z^{2}$ whereas for $z \gg 1, u_{0} \simeq 1-\mathrm{e}^{-z}$ and $s_{0} \simeq-r \mathrm{e}^{-z}$. These results imply that the survival probability decays asymptotically as

$$
\begin{aligned}
& Q\left(x_{0}, t\right) \simeq 2 z^{2} \exp \left(-r t\left(1-z^{2}+\ldots\right)\right) \text { for } z \ll 1 \\
& Q\left(x_{0}, t\right) \simeq \exp (-r t \exp (-z)) \text { for } z \gg 1
\end{aligned}
$$

One may relate the large $z$ result to the Gumbel distribution for the extremum of independent random variables [11] as follows. In time $t$ the mean number of resets to position $x_{0}$ is $N=r t$. For the target to survive requires that between each reset the maximum distance to the left attained by the particle is less than $x_{0}$. In a given reset interval of length $\tau$, the particle undergoes free diffusion around $x_{0}$. Hence, the probability that the minimum is bigger than $m$ is erf $\left(\frac{\left|m-x_{0}\right|}{\sqrt{4 D \tau}}\right)$ [1] and the pdf of the minimum $m$ is $\operatorname{Prob}(m)=e^{-\left(m-x_{0}\right)^{2} / 4 D \tau} / \sqrt{\pi D \tau}$ with $m \leq x_{0}$. Next, we average over $\tau$ drawn from the exponential distribution $\operatorname{Prob}(\tau)=r e^{-r \tau}$. Thus, the effective pdf of the maximum over each reset interval is then $p(m)=\alpha_{0} e^{-\alpha_{0}\left(x_{0}-m\right)}$ where $m \leq x_{0}$. Taking the $N$ intervals as statistically independent, the probability that the minimum of all $N$ intervals stays above 0 is $\left[\int_{0}^{x_{0}} p(m) d m\right]^{N}$. Performing the integral and taking large $z$ limit yields $\approx \exp [-r t \exp (-z)]$.

We now turn to the survival probability of a stationary target at the origin in the presence of many independent searchers (diffusive particles). We consider $N$ diffusive particles $i=1, \ldots, N$, each of which is reset independently to its initial position $x_{i}$ with rate $r$. The survival probability of the target is given by

$$
P_{s}(t)=\prod_{i=1}^{N} Q\left(x_{i}, t\right)
$$

where $Q\left(x_{i}, t\right)$ is the survival probability in the single searcher problem.

The initial positions $x_{i}$ 's are assumed to be independent and each distributed uniformly over the box $[-L / 2, L / 2]$. Consequently, $P_{s}(t)$ is a random variable. Its average is simply $P_{s}^{\text {av }}(t)=\left\langle P_{s}(t)\right\rangle_{x}$ where \langle\rangle$_{x}$ denotes averages over $x_{i}$ 's. However, $P_{s}(t)$ for a typical initial configuration is not captured by the average. The typical $P_{s}(t)$ can be extracted by first averaging over the logarithm of $P_{s}(t)$ followed by exponentiating: $P_{s}^{\text {typ }}(t)=\exp \left[\left\langle\ln P_{s}(t)\right\rangle_{x}\right]$. One can draw an analogy to a disordered system with $P_{s}(t)$ playing the role of partition function $Z$ and $x_{i}$ 's as disorder variables. Thus the average and typical behavior correspond respectively to the annealed (where one averages the partition function $Z$ ) and the quenched (where one averages the free energy $\ln Z$ ) averages in disordered systems.

In the annealed case we get

$$
P_{s}^{\mathrm{av}}(t)=\langle Q(x, t)\rangle_{x}^{N}=\exp N \ln \left[1-\langle 1-Q\rangle_{x}\right]
$$

where

$$
\langle 1-Q\rangle_{x}=\frac{1}{L} \int_{-L / 2}^{L / 2} \mathrm{~d} x[1-Q(x, t)] .
$$

Letting $N, L \rightarrow \infty$ but keeping the density of walkers $\rho=$ $N / L$ fixed, and using the symmetry $Q(x, t)=Q(-x, t)$, we obtain

$$
P_{s}^{\mathrm{av}}(t) \rightarrow \exp -2 \rho \int_{0}^{\infty} \mathrm{d} x[1-Q(x, t)] \equiv \exp -2 \rho M(t) .
$$


The Laplace transform $\tilde{M}(s)=\int_{0}^{\infty} M(t) e^{-s t} d t$ can be determined using (6)

$$
\tilde{M}(s)=\frac{r+s}{s r \alpha} \ln \left(\frac{s+r}{s}\right) .
$$

It may then be inverted [12] to obtain

$$
M(t)=\left(\frac{D}{r}\right)^{1 / 2} \mu(y),
$$

with $y=r t$, where

$$
\mu(y)=\int_{0}^{y} \mathrm{~d} v \frac{1-\mathrm{e}^{-v}}{v}\left[\operatorname{erf}\left[(y-v)^{1 / 2}\right]+\frac{\mathrm{e}^{-(y-v)}}{\sqrt{\pi(y-v)}}\right] .
$$

The function $\mu(y)$ behaves asymptotically as

$$
\begin{aligned}
\mu(y) & \simeq \frac{2 y^{1 / 2}}{\sqrt{\pi}} \quad \text { for small } y \\
& \simeq \ln y+\gamma \quad \text { for large } \quad y
\end{aligned}
$$

where $\gamma$ is Euler's constant. Thus the long-time behaviour $(r t \gg 1)$ of the annealed survival probability is a power law with an exponent that varies continuously with the density (17) as

$$
P_{s}^{\mathrm{av}}(t) \simeq A t^{-2 \rho(D / r)^{1 / 2}},
$$

where $A$ is a constant that may be determined. The short-time behaviour $(r t \ll 1)$ is stretched exponential decay $P_{s}^{\mathrm{av}}(t) \simeq \exp \left(-4 \rho(D t)^{1 / 2} / \sqrt{\pi}\right)$ as in the case of diffusion without resetting [9, 10].

In contrast, the typical behavior (the quenched case) $P_{s}^{\text {typ }}(t)=\exp \left[\left\langle\ln P_{s}(t)\right\rangle_{x}\right]$ can be expressed as

$P_{s}^{\text {typ }}(t)=\exp \sum_{i=1}^{N}\left\langle\ln Q\left(x_{i}, t\right)\right\rangle_{x}=\exp \left[2 \rho \int_{0}^{L / 2} \mathrm{~d} x \ln Q(x, t)\right]$

In the long time limit, we have using (11)

$$
\int_{0}^{\infty} \mathrm{d} x_{0} \ln Q\left(x_{0}, t\right) \simeq \text { Constant }\left[-t \int_{0}^{\infty} \mathrm{d} x_{0}\left|s_{0}\left(x_{0}\right)\right|\right] .
$$

The integral can be done in closed form [12] and we find $\int_{0}^{\infty} \mathrm{d} x_{0} s_{0}\left(x_{0}\right)=-(D r)^{1 / 2} 4(1-\ln 2)$. Thus the asymptotic decay of the quenched total survival probability is exponential

$$
P_{s}^{q}(t) \sim \exp \left(-t \rho(D r)^{1 / 2} 8(1-\ln 2)\right) .
$$

The correction to the argument of the exponential in (26) will come from the branch point at $s=-r$ in (6) and is expected to give a contribution $O\left(t^{1 / 2}\right)$. The fact that the average and typical survival probabilities have distinct asymptotic behaviours reflects the strong dependence on the initial conditions whose memory is retained through resetting.

Our results can straightforwardly be extended to higher dimensions. For example, in $d$-dimensions, the second derivative term in the Master equation (11) is replaced by a $d$-dimensional Laplacian. The stationary solution in $d$-dimensions is easy to find [12]

$$
p_{\mathrm{st}}\left(\vec{x} \mid \vec{x}_{0}\right)=\frac{\left(\alpha_{0}\right)^{d}}{(2 \pi)^{d / 2}}\left(\alpha_{0}\left|\vec{x}-\vec{x}_{0}\right|\right)^{\nu} K_{\nu}\left(\alpha_{0}\left|\vec{x}-\vec{x}_{0}\right|\right)
$$

where $\nu=1-d / 2$ and $K_{\nu}$ is the modified Bessel function. Similarly, the mean absorption time for an absorbing ball of radius $\epsilon$ at the origin is [12]

$$
T\left(\vec{x}_{0}\right)=\frac{1}{r}\left[\left(\frac{\epsilon}{\left|\vec{x}_{0}\right|}\right)^{\nu} \frac{K_{\nu}\left(\alpha_{0} \epsilon\right)}{K_{\nu}\left(\alpha_{0}\left|\vec{x}_{0}\right|\right)}-1\right] .
$$

The survival probability in case of multiple searchers, both annealed and quenched, can also be computed in higher dimensions [12].

In conclusion, in this Letter we have shown that introduction of resetting events leads to rather rich effects on simple diffusion process. In particular, the effect of resetting on the efficient search for a stationary target both by a single or a team of searchers is rather profound. Our results can be extended in several directions, in particular one may consider resetting rates $r(x)$ that depend on the position of the particle [12]. It would also be interesting to study the effect of resetting on other available search strategies based on nondiffusive processes such as Lévy flights [4, 5].

We thank R. A. Blythe, B. Derrida and S. Janson for useful discussions.

·1] S. Redner A Guide to First-Passage Processes (Cambridge University Press, Cambridge 2001).

[2] S. N. Majumdar, Curr. Sci. 77, 370 (1999).

[3] W.J. Bell, Searching Behaviour (Chapman and Hall, London) (1991).

[4] O. Bénichou, M. Coppey, M. Moreau, P-H. Suet, and R. Voituriez Phys. Rev. Lett.94, 198101 (2005)

[5] M. A. Lomholt, T. Koren, R. Metzler, J. Klafter P. N. A. S. 105, 11055 (2008)

[6] C. Loverdo, O. Bénichou, M. Moreau, and R. Voituriez Phys. Rev. E80, 031146 (2009)

[7] F. Bartumeus and J Catalan J. Phys. A: Math. Theor. 42, 434002 (2009).

[8] G. Oshanin, K. Lindenberg, H. S. Wio and S. Burlatsky J. Phys. A: Math. Theor.42, 434009 (2009)

[9] A. Blumen, G. Zumofen, and J. Klafter, Phys. Rev. B 30, 5379 (1984).

[10] A. J. Bray and R. A. Blythe, Phys. Rev. Lett. 89, 150601 (2002) and references therein.

[11] E. J. Gumbel, Statistics of Extremes (Columbia University Press, New York, 1958).

[12] details will be published elsewhere. 